\title{
Anti-neutrino measurements in SNO+
}

\author{
Sofia Andringa ${ }^{* \dagger}$ \\ LIP - Laboratório de Instrumentação e Física Experimental de Partículas \\ E-mail: sofia@lip.pt
}

$\mathrm{SNO}+$ is a new neutrino physics experiment, that will start collecting data in 2016, reusing the SNO detector with 780 tons of liquid scintillator as active medium. It will perform several low energy measurements, namely the search for neutrinoless double beta decay, for which the scintillator will be loaded with tellurium. Anti-neutrino interactions can be identified through a characteristic delayed coincidence signature, between a positron annihilation and a neutron capture. SNO+ will measure anti-neutrinos from nuclear reactors, useful for oscillation studies, and geoneutrinos, useful for Geophysics studies. The reactor and geo-neutrinos have very different energy spectra, which is the main variable to disentangle them. We explore the possibility to use time variations of the expected fluxes and some direction sensitivity to increase the separation between the anti-neutrino sources, for example between geo-neutrinos from crust and mantle.

The main advantage of SNO+ for the anti-neutrino analysis is that the flux is dominated by reactors in two different directions and two distances of the order of $100 \mathrm{~km}$, which give rise to sharp spectral features in the oscillated energy spectrum and a high sensitivity to the neutrino squared mass difference parameter. On the other hand, the small number of near-by reactors implies also a small background for the geo-neutrino measurement, to be done in a new geological location.

The European Physical Society Conference on High Energy Physics

22-29 July 2015

Vienna, Austria

* Speaker.

${ }^{\dagger}$ for the SNO+ Collaboration 


\section{The SNO+ experiment}

SNO+ will reuse most of the hardware from the SNO detector. It is located at SNOLAB in Canada, at a depth of $2000 \mathrm{~m}$, and has 9300 PMTs observing the inside of a $12 \mathrm{~m}$ diameter sphere made of transparent acrylic, surrounded by ultra-pure water for further radiation shielding. The active medium will consist of 780 tons of liquid scintillator, allowing for a low energy threshold of the order of $0.1 \mathrm{MeV}$.

The main physics goal of the experiment is the search for neutrinoless double beta decay of Te-130, for which a load of $0.3 \%$ of natural Tellurium will be dissolved in the liquid scintillator. The corresponding decay in the two neutrinos mode will produce the largest rate physics signal, and with the tellurium load, extra internal backgrounds will arise. The energy resolution will be slightly degraded down to $6 \%$ or $7 \%$ at $1 \mathrm{MeV}$. A shorter data taking period will be done without the tellurium, resulting in less background and higher resolution.

In the next year, SNO+ will do a commissioning run in water. The tagging of anti-neutrinos, with the detection of a sub-threshold signal of the $2.2 \mathrm{MeV}$ gamma coming from a neutron capture will be attempted in that phase also. In the loaded and un-loaded scintillator phase, the $2.2 \mathrm{MeV}$ gamma is well above threshold and gives a very distinctive signal for anti-neutrino studies.

Further details on the detector, general physics plans and status of the SNO+ experiment are described in [1] and in another contribution to these proceedings [2].

\section{Anti-neutrino sources and oscillations}

Around 110 anti-neutrino interactions per year are expected in $\mathrm{SNO}+$, most of them generated by nuclear reactors but also other by the Earth's natural radioactive chains of uranium and thorium. Neutrino oscillations will distort the known spectrum of electron anti-neutrinos from the reactors, while the effect for geo-neutrinos will be averaged due to the large ranges of distances to be considered.

The electron anti-neutrino survival probability is determined by the oscillation parameters as:

$$
P_{e e} \sim \cos ^{4} \theta_{13}\left(1-\sin ^{2}\left(2 \theta_{12}\right)\left(1.27 \Delta m_{12}^{2} L / E\right)\right)^{2}
$$

Given the present values of the oscillation parameters, and namely of $\Delta m_{12}^{2} \sim 0.2 \times 10^{-5} \mathrm{eV}$ [3], the oscillation pattern becomes very clear for fixed distances (L) of the order of $100 \mathrm{~km}$ and energies (E) of some $\mathrm{MeV}$ as available in reactors. With a flux dominated by two different baseline distances, of $250 \mathrm{~km}$ and $350 \mathrm{~km}$, SNO+ will obtain a very clear oscillation pattern as shown in figure 1.

Geo-neutrinos originate in the crust and mantle, spanning a large range of distances, and so the oscillation pattern averages out, leaving only a global decrease of the observable flux. They are created by beta decays in the uranium and thorium chains, and can thus be related to the energy released by those chain decays inside the Earth. While most of the geo-neutrinos are below the energy threshold of current experiments, those of the uranium chain extend up to $3.3 \mathrm{MeV}$ and those of the thorium chain to $2.3 \mathrm{MeV}$. These have been previously measured by both KamLAND [4], in Japan, and Borexino [5], in Italy. SNO+ will provide a third measurement of this important probe 


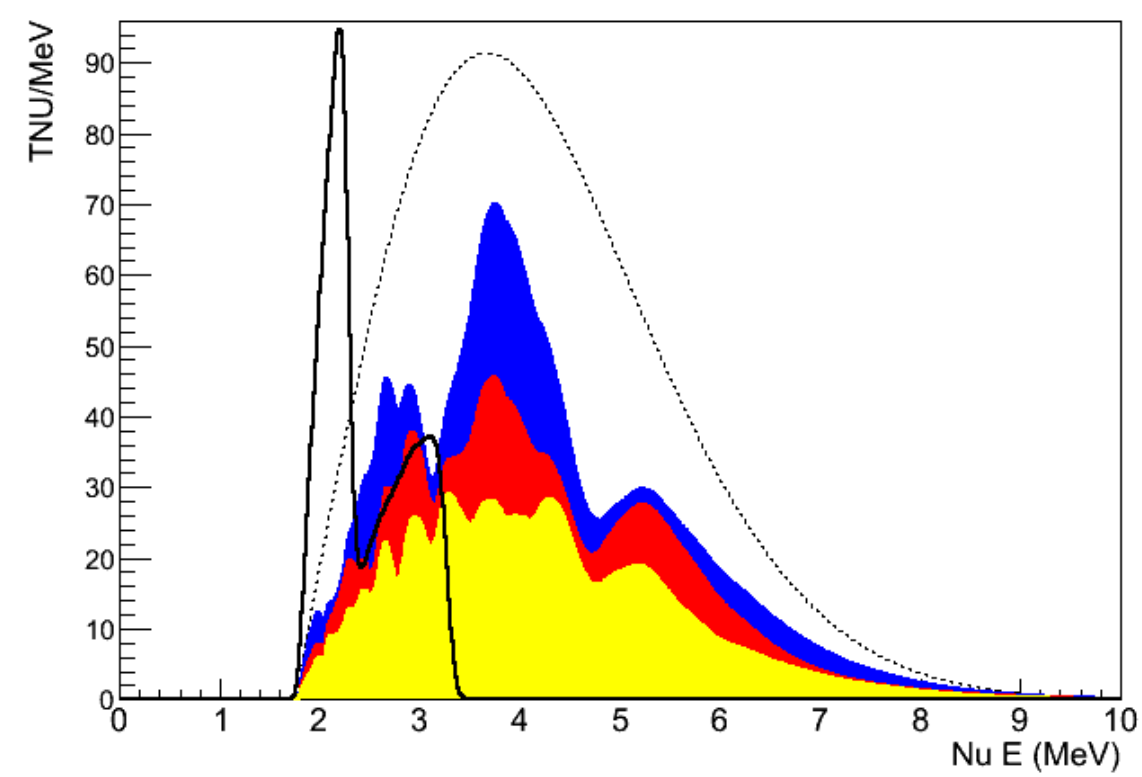

Figure 1: Expected visible anti-neutrino energy spectrum in $\mathrm{SNO}+$, in Terrestrial Neutrino Units, defined as $10^{32}$ protons-year, per MeV. The dashed line represents the non-oscillated reactor spectrum, the colored region is the oscillated reactor spectrum, divided in the contribution of the closest reactor complex (in blue), the two other Canadian reactors (in red) and the extra reactor contributions (in yellow), from top to bottom. The full line represents the geo-neutrino spectrum, with an arbitrary normalization.

for geo-sciences, in a different location, with high crustal contribution, in order to give a better estimate of the global radiogenic heat flow and test the mantle composition models.

In addition to the energy spectrum, which allows to distinguish the reactor flux from the geoneutrino flux, we will try to explore both the time variations of the fluxes and the incoming direction of the anti-neutrinos. The geo-neutrino contribution is constant in time, with predominantly upward directions from the mantle and mostly horizontal from the crust; the reactor output variations are well monitored, and each one is at a fixed azimuthal direction. Notice, however, that contrary to the anti-neutrino energy and interaction time which can be reconstructed with high precision, the direction is in general not reconstructed, at least in an event-by-event case. Due to the very particular distribution of sources, we will explore the possibility to retain some statistical distinction.

\section{Anti-neutrino detection and reconstruction}

Anti-neutrinos will interact through the so-called inverse beta decay process, $\bar{v} p \rightarrow e^{+} n$, implying a minimum energy of $1.8 \mathrm{MeV}$ to get the neutron to proton mass difference plus the positron mass. The positron will carry the rest of the available energy, and will annihilate immediately, the total energy of this prompt event (with a minimum at twice the electron mass) is linearly correlated to the anti-neutrino energy. The neutron will be correlated with the direction of the anti-neutrino, at an average angle of 25 degrees for the low energy geo-neutrinos, and 40 degrees for the higher energy reactor anti-neutrinos. 
The neutron will scatter inside the detector until it thermalizes and is captured by a free proton at a different location. This will create an excited state of deuterium which will emit a 2.2 $\mathrm{MeV}$ gamma isotropically. The gamma will be detected only after an inverse Compton (or pair production) interaction adding a further distance from the prompt interaction.

The distance in time and position between the prompt positron and the capture gamma detection events are given by exponentials with characteristic lengths of $200 \mu \mathrm{s}$ and $0.3 \mathrm{~m}$. The relaxed coincidence between the two events, with $1 \mathrm{~ms}$ time difference and $1 \mathrm{~m}$ distance are enough to reduce most backgrounds to negligible levels.

The information about the initial neutron direction is contained on the vector between the two events, but is heavily smeared except for a very small sample of events in which the scattering of the neutral particles inside the detector is reduced by tight cuts on both variables.

\section{Analysis and expected results}

The properties of neutron scattering and capture have been simulated with a full detector simulation based in Geant 4 and will be later calibrated with an AmBe source generating real coincidences between a neutron and a prompt high energy gamma signal through $(\alpha, n)$ interactions.

The main background concern comes from similar high energy $(\alpha, n)$ reactions, in which the scattering of protons (and $\alpha$ ) can give prompt signals correlated to those from the neutron capture. A possible source of those events inside the detector is the alpha decay of ${ }^{210} \mathrm{Po}$, with a subsequent $\alpha$ interaction on ${ }^{13} \mathrm{C}$ producing a neutron (and a ${ }^{16} \mathrm{O}$ nucleus).

In fact, some polonium may have accumulated in the acrylic vessel wall as the detector was opened in the previous years, and can leach into the scintillator. This kind of background will be quantified at the beginning of data taking in a pure-scintillator run, and prompt gamma peaks associated to the same process will be used to study their evolution in time. In any case, the proton and $\alpha$ scattering signals are quenched to very low energies, and concentrate in the low energy region, affecting the geo-neutrinos measurement more than the oscillation measurements.

Preliminary estimates show that by fitting the energy spectrum above $3.5 \mathrm{MeV}$, using the expected energy resolution of $6 \%$ with the tellurium loading, $\mathrm{SNO}+$ will be able to measure $\Delta m_{12}^{2}$ with a precision equivalent to the current existing one, of $0.2 \times 10^{-5} \mathrm{eV}^{2}$, in seven years. The same result can be achieved earlier by extending the analysis to the full energy range. For the final analysis, an accurate modeling of the geo-neutrino and $(\alpha, n)$ backgrounds will be needed. The variations in time of the several sources, the radial position inside the detector (to decrease external backgrounds) and the characteristic difference of the coincident events in time and distance (which can eventually lead to direction sensitivity) must also be included.

SNO+ will attempt to measure also the separate fluxes of geo-neutrinos, ie, the thorium and uranium chain contributions, which can be separated through the energy spectrum, and the crust and mantle, for which direction sensitivity could be a very useful tool. Even if this is not achievable due to the low statistics, a full combination with external information about the nearby crust and with the geo-neutrino spectrum measured by the other experiments will provide useful results for geo-sciences.

In fact, geo-neutrinos are becoming a new tool for geo-sciences and can provide constraints to the Earth modeling. The energy released by a kilogram of a given element can be directly related 
to the rate of geo-neutrinos emitted $(950 \mathrm{~mW}: 750 \bar{v} / \mu$ s for uranium and $270 \mathrm{~mW}: 162 \bar{v} / \mu$ s for thorium), and even though only a few are detectable, they give a direct constraint to the presence of these elements in the inner Earth, which can not be sampled directly in other ways.

\section{References}

[1] SNO+ Collaboration, "Current Status and Future Prospects of the SNO+ Experiment", Submitted to the special issue "Neutrino Masses and Oscillations" of Advances in High Energy Physics (Hindawi), arXiv:1508.05759 [physics.ins-det], (2015)

[2] G. Prior, "Status of the SNO+ experiment", these proceedings

[3] KamLAND Coll., Phys. Rev. Lett. 100 221803, 2008;

[4] KamLAND Coll., Nature Geoscience 4 647, 2011;

[5] Borexino Coll. Phys. Lett. B 722 295, 2013; 